\title{
Infantile Acute Subdural Hematoma with Retinal Hemorrhage Caused by Minor Occipital Impact Witnessed by an ICU Nurse: A Case Report
}

\author{
Nobuhiko Aoki, M.D..$^{1,2^{*}}$ \\ ${ }^{1}$ Department of Neurosurgery, Tokyo Metropolitan Tama Medical Center, Japan \\ ${ }^{2}$ Bethlehem Garden Hospital, Japan
}

\begin{abstract}
An acute subdural hematoma (ASDH) in infants without external signs of trauma is differently recognized as shaken baby syndrome (SBS) or abusive head trauma (AHT) in the United States or as infantile acute subdural hematoma (i-ASDH) due to minor head trauma in Japan. The aim of the present case report is to document the existence of $\mathrm{i}-\mathrm{ASDH}$ as a discrete clinical entity differing from SBS and AHT.

A 7-month-old male suffered a subdural hematoma and retinal hemorrhage after falling backwards from a sitting position while at home. Although the patient regained full activity in the emergency room, he was hospitalized for further observation. Six days after admission, the patient was witnessed by an ICU nurse falling backwards from a seated position on his bed and striking his occipital region against the bed railing. He began to cry and soon afterwards exhibited conjugate eye deviation, cyanosis, rigidity of the extremities, and loss of consciousness. An emergency CT revealed mixed-density ASDH, and an ophthalmologic examination disclosed multiple multilayered retinal hemorrhages. This case report provides clear evidence proving that ASDH in infants can be caused by short falls.
\end{abstract}

\section{Keywords}

Abusive head trauma, Accidental head trauma, Infantile acute subdural hematoma, Retinal hemorrhage, Shaken baby syndrome

\section{Introduction}

An acute subdural hematoma (ASDH) accompanied by a retinal hemorrhage $(\mathrm{RH})$ is often regarded without question worldwide as indicative of shaken baby syndrome (SBS)/ abusive head trauma (AHT). However, this tendency is based exclusively on the assumption that short falls are unable to cause ASDH [1].

Because infantile ASDH (i-ASDH), mostly reported in Japan [2], rarely occur outside the home, the accidents leading to the trauma are seldom witnessed by a medically-trained third party. The author herein discusses the case of an infant who struck his occipital region in a short fall during hospitalization and suffered ASDH with RH. The entire accident was witnessed by an ICU nurse. The present case provides evidence of accidental ASDH occurring after mild head trauma as reported previously in Japan in more than 200 cases [2].

\section{Case Presentation}

A 7-month-old male with no significant medical history fell backwards from a seated position and struck his occipital region on the carpeted floor inside his home in November 2017. The accident was witnessed by his parents, who happened to be in front of the patient. The infant began crying imme- diately. His father held him in his arms and noted an upward deviation of both eyes, cyanosis, flaccidity, and altered consciousness. The infant was transferred to a national hospital on an emergency basis. En route, while in the ambulance, he regained full activity and returned to his baseline.

In the emergency room, a pediatrician identified thin ASDH with the maximum thickness of $2 \mathrm{~mm}$ on CT (Figure 1) and decided to admit the patient for continued observation. No neurological abnormalities or external signs of trauma were noted. A full skeletal survey, including a 3-D cervical spinal $\mathrm{CT}$, revealed no abnormalities. A hematologic examination

*Corresponding author: Nobuhiko Aoki, M.D, Department of Neurosurgery, Tokyo Metropolitan Tama Medical Center, 1838524, 3-14-72 Musashidai , Fuchu-shi, Tokyo, Japan, Tel: +8142-323-5111, Fax: +81-42-323-9205

Accepted: March 19, 2020

Published online: March 21, 2020

Citation: Aoki N (2020) Infantile Acute Subdural Hematoma with Retinal Hemorrhage Caused by Minor Occipital Impact Witnessed by an ICU Nurse: A Case Report. J Pediatr Neurol Neurosci 4(1):47-50 
was unremarkable and showed no thrombocytopenia or other hemorrhagic diathesis. Two days after admission, a fundoscopic examination disclosed bilateral RH (Figure 2).

Although his clinical course was unremarkable, a follow-up CT showed an increase in the ASDH on Day 3, followed by a rapid decrease on Day 4 (Figure 3 ). He was doing well until Day 6, when he again fell backwards from a seated position while on a circular bed for children in the ICU and struck his occipital region against the metallic bed rail. The accident was

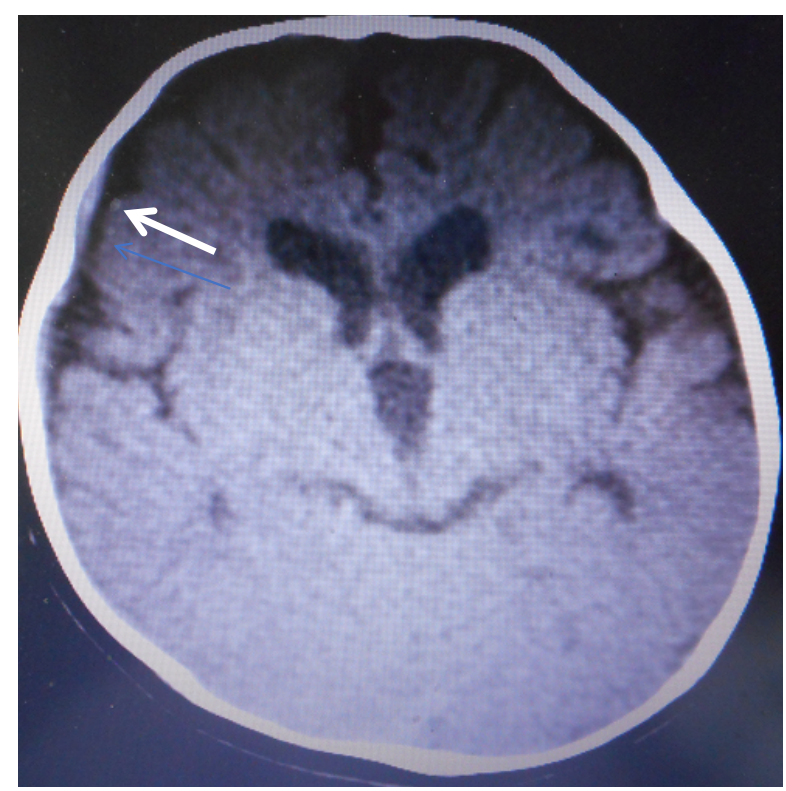

Figure 1: CT on the day of injury, showing a thin, high-density subdural hematoma with the maximum thickness of $2 \mathrm{~mm}$ at the right convexity (arrow). Note the association of the enlarged subarachnoid spaces and ventricles. directly witnessed by an ICU nurse, who documented the incident as below:

"Soon after a mild occipital impact against the bed rail, the patient began crying, then exhibited conjugate eye deviation, cyanosis, rigidity of the extremities, and loss of consciousness. His pulse rate rapidly decreased to 60 beats per minute but returned to the baseline of 110 beats six minutes later".

Immediately after the onset of neurological deterioration, he was intensively managed with anesthetic and anticonvulsant administration under full oxygenation.

An emergency CT showed mixed-density ASDH (Figure 4), and an ophthalmologic examination revealed bilateral multiple, multilayered, retinal hemorrhages (Figure 5).

The ASDH, which was not surgically removed, gradually decreased in size. Five days later, he showed good recovery without neurological deficits and has not experienced any recurrence of the symptoms.

An MRI performed 14 days after the injury revealed no abnormality in the cerebral parenchyma apart from the residual SDH. The patient had achieved normal developmental milestones at the time of his last examination two years later.

\section{Discussion}

More than thirty years have passed since the publication of "Infantile acute subdural hematoma: Clinical analysis of 26 cases" by Aoki and Masuzawa in the Journal of Neurosurgery [3]. Based on the anatomical characteristics of infants, the authors restricted their definition of infantile acute subdural hematoma (i-ASDH) to an acute subdural hematoma apparently caused by minor head trauma without loss of consciousness or primary brain injury [3]. At the time, the authors were criti-

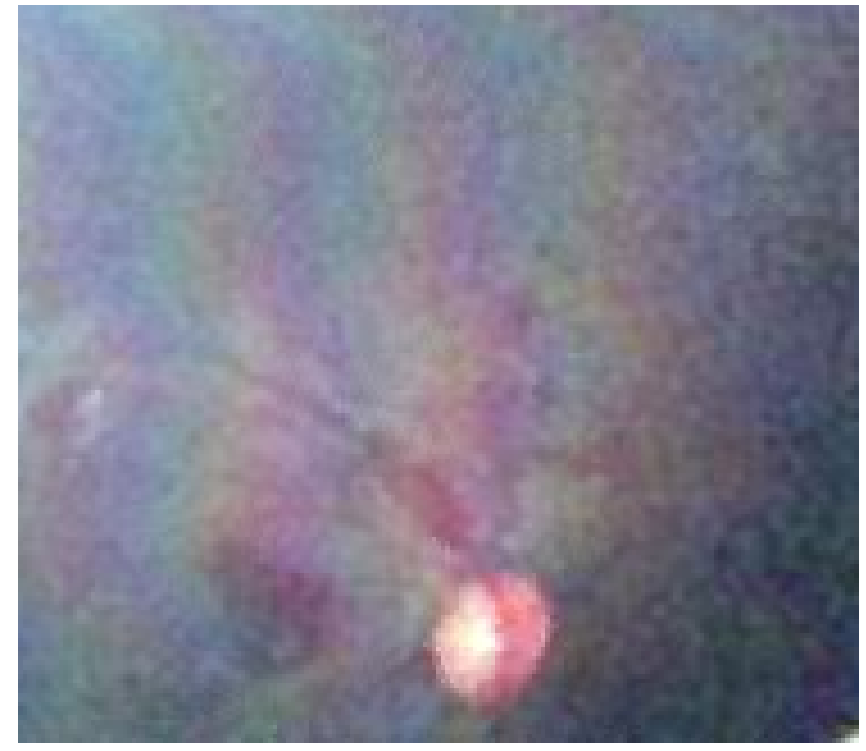

(A)

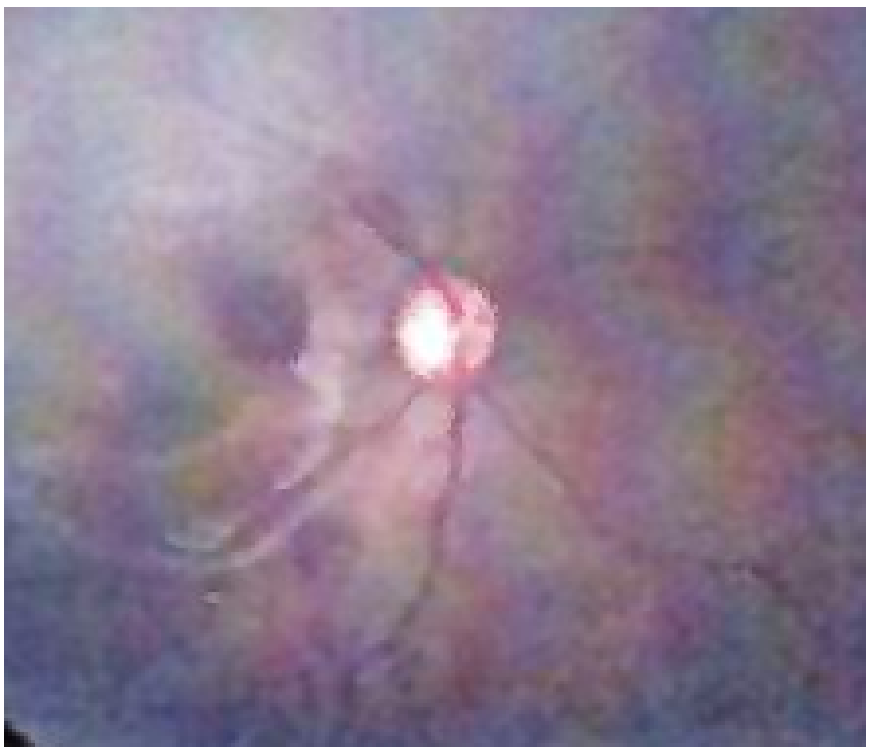

(B)

Figure 2: Fundoscopic examination on Day 2 showing mild retinal hemorrhages at the bilateral occipital poles.

A) Left side; B) Right side. 


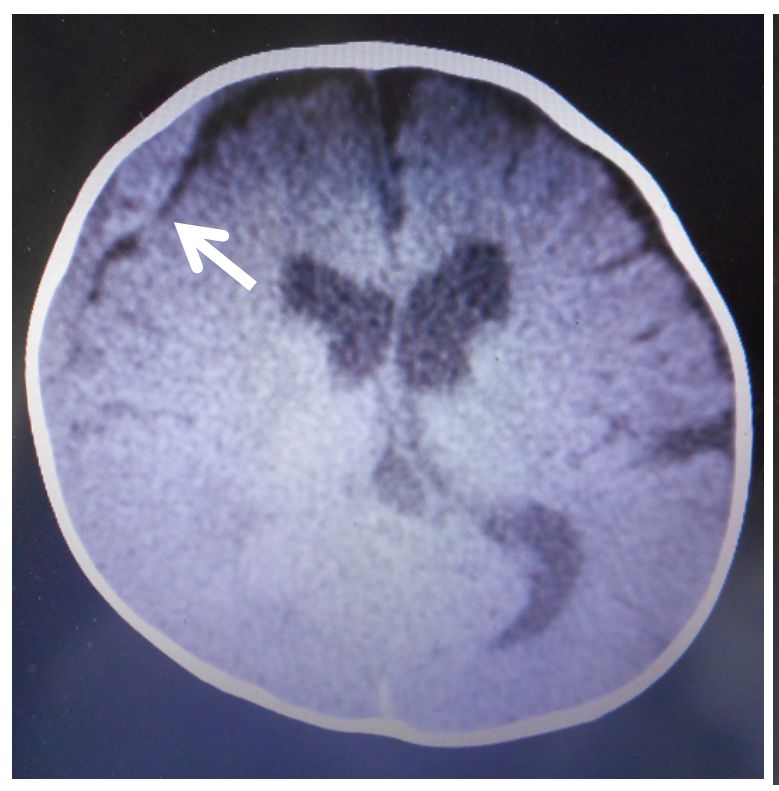

(A)

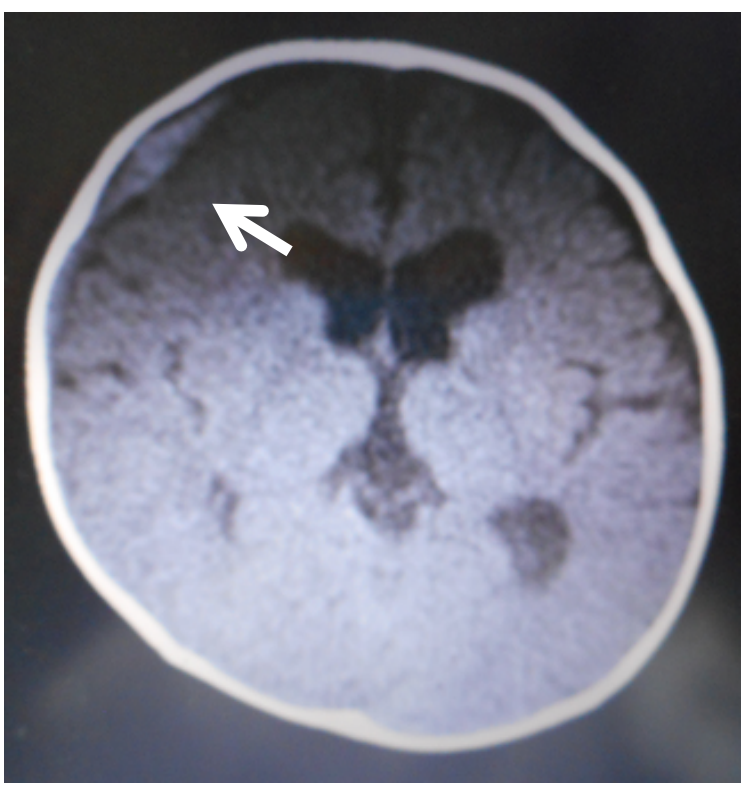

(B)

Figure 3: Follow-up CT. An increase in the size of the high-density subdural hematoma is noted on Day 3 (arrow), and a decrease is shown on Day 4 (arrow). A) CT on day 3; B) CT on day 4.

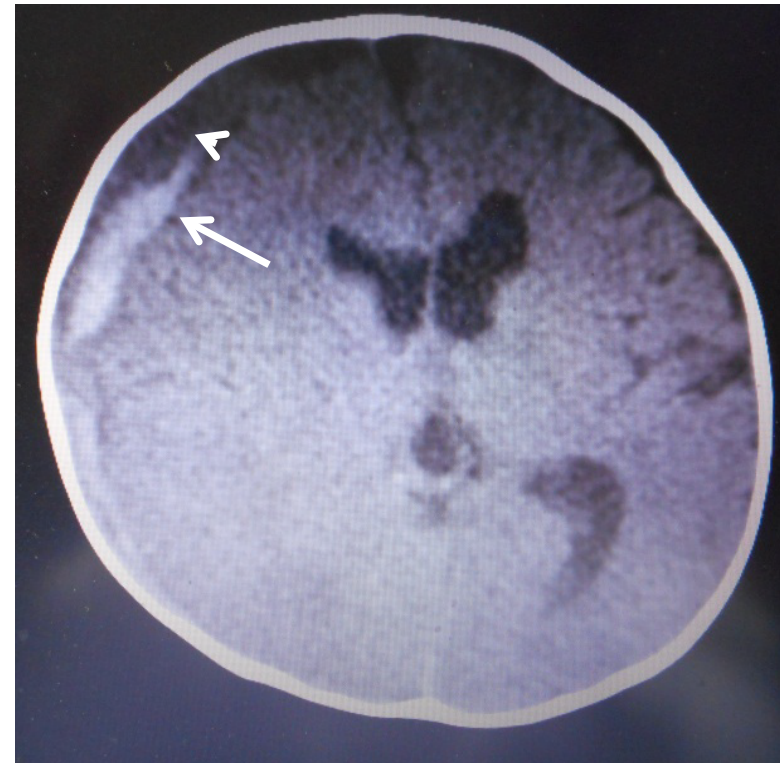

Figure 4: CT on Day 6 immediately after the accident demonstrating an increase in the size of the high-density subdural hematoma (arrow) and low-density subdural hematoma (arrowhead).

cized for allegedly failing to recognize that most, if not all, the patients cited in their study had suffered whiplash in shaken baby syndrome and were the victims of child abuse [1]. The constellation of SDH and retinal hemorrhage in the context of minor trauma, while not necessarily pathognomonic of trauma from shaking, should be regarded as symptoms of whiplash in shaken baby syndrome unless another etiology can be determined.

Partly due to this criticism, the authors' suggestion that
i-ASDH may be an independent clinical entity has not been fully accepted, at least in the English-speaking world. In Japan, however, since more than 30 -years ago, mild head trauma has been recognized as the most frequent cause of ASDH in infants. The current estimate of its incidence in Japan is approximately 120 cases per year [4].

Infantile ASDH is not widely recognized in the United States and other nations because reports of it originate mostly in Japan. In the United States, i-ASDH might be diagnosed as SBS/AHT due to its rarity and the historical precedent for the diagnosis of ASDH in infants.

Reports of i-ASDH from Japan include 14 articles covering more than 200 cases [2]. All but two of these articles [3,5] were published in Japanese, with the result that their findings remain largely unknown in the United States.

There are three, distinct differences between $\mathrm{i}-\mathrm{ASDH}$ and SBS/AHT. First, i-ASDH generally has a benign clinical course, with approximately $80 \%$ of patients showing good recovery $[1,3,6-8]$. Second, i-ASDH is strictly age-specific and occurs mostly in male infants between 6 and 12 months old. As a result, accidents occurring outside the home are extremely rare and seldom witnessed by a third party. Recently in the United States, SDH was diagnosed in eight patients younger than 24 months who were witnessed by family members suffering a fall that resulted in an occipital impact [9]. In the present case series as well, the witnesses were family members, relatives or children, and no medically trained personnel were present as witnesses.

Third, and most importantly, the fundamental aspect of $\mathrm{i}$-ASDH pathology distinguishing it from SBS/AHT is the absence of a primary cerebral parenchymal injury, as confirmed by imaging studies and surgery. These pathological features 
Citation: Aoki N (2020) Infantile Acute Subdural Hematoma with Retinal Hemorrhage Caused by Minor Occipital Impact Witnessed by an ICU Nurse: A Case Report. J Pediatr Neurol Neurosci 4(1):47-50

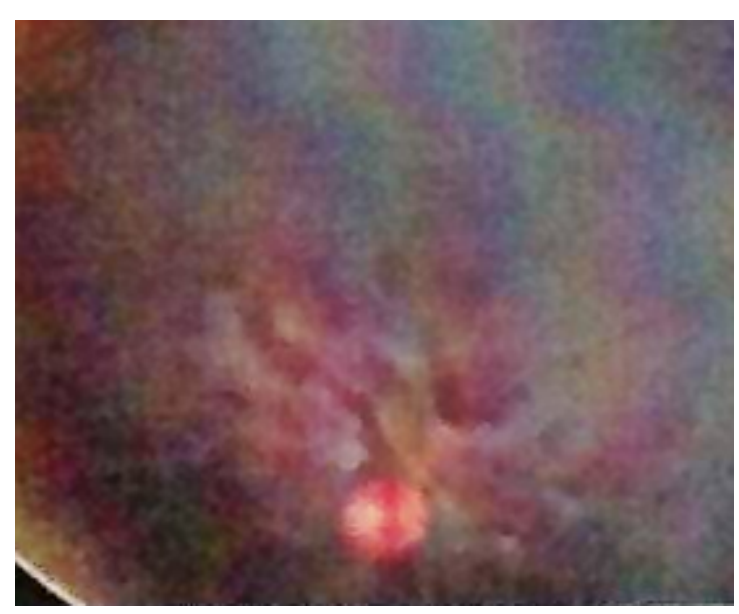

(A)

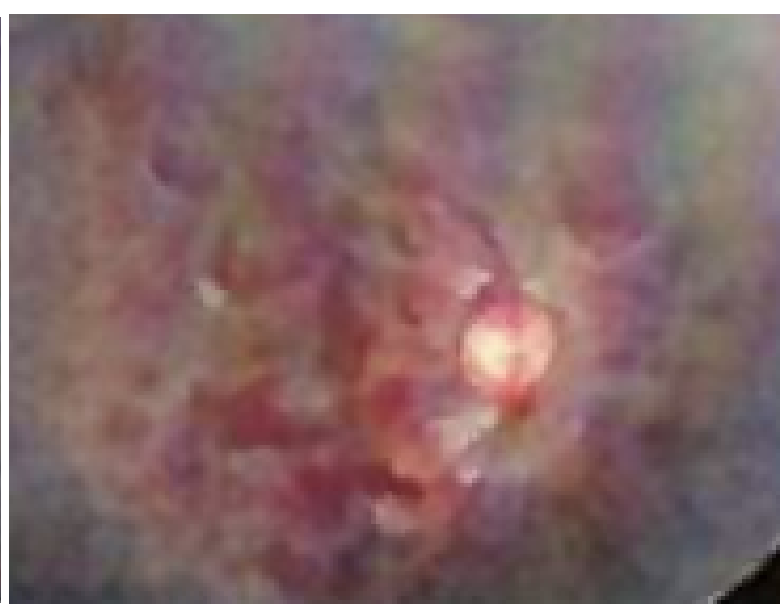

(B)

Figure 5: Fundoscopic examination on Day 6 after the accident, showing newly developed bilateral multiple, multilayered retinal hemorrhages. A) Left side and B) Right side.

of i-ASDH, including the fulminant type, are compatible with its mostly benign clinical course following conservative management or rapid surgical intervention [3]. In addition, the present case differs from those cited by Plunkett in his case series [10] in terms of age, clinical outcome, and the absence of primary brain injury.

Although multilayered retinal hemorrhages are considered to be highly suggestive of child abuse [11], the present case confirmed that retinal hemorrhages identical to those observed in the victims of child abuse are also associated with i-ASDH.

\section{References}

1. Rekate $H$ (1985) Subdural hematomas in infants. J Neurosurg 61: 316-317.

2. Aoki N (2011) Infantile acute subdural hematoma: Caused by abuse or minor head trauma? Nervous System in Children 36: 326-230.

3. Aoki N, Masuzawa H (1984) Infantile acute subdural hematoma. Clinical analysis of 26 cases. J Neurosurg 61: 273-280.
4. Aoki N (2017) Letter to the editor. Nervous System in Children 42: 393-397.

5. Ikeda A, Sato O, Tsugane R, et al. (1987) Infantile acute subdural hematoma. Childs Nerv Syst 3: 19-22.

6. Nishimoto $\mathrm{H}$, Kurihara J (2006) Re-estimation of acute subdural hematoma in children caused by trivial household head trauma. Nervous System in Children 31: 215-223.

7. Park YS, Kotani Y, Sugimoto T, et al. (2010) Clinical comparative analysis of abusive injuries and accidental injuries in infantile subdural hematoma. Nervous System in Children 38: 354-363.

8. Yamasaki M, Nonaka M (2009) Clinical characteristic findings and treatment of pediatric abusive head injury: Key points for neurosurgeons. Jpn J Neurosurg (Tokyo) 18: 64264-64269.

9. Atkinson N, Van Rijin RR, Starling SP (2018) Childhood falls with occipital impacts. Pediatr Emerg Care 34: 837-841.

10. Plunkett J (2001) Fatal pediatric head injuries caused by short-distance falls. Am J Forensic Med Pathol 22: 1-12.

11. Morad Y, Wygnanski-Jaffe T, Levin AV (2010) Retinal haemorrhage in abusive head trauma. Clin Exp Ophthalmol 38: 514-520.

DOI: $10.36959 / 595 / 406$ 